\title{
Local Identifiability and Observability of Nonlinear Differential-Algebraic Equations
}

\author{
Markus Gerdin \\ Division of Automatic Control \\ Department of Electrical Engineering \\ Linköpings universitet, SE-581 83 Linköping, Sweden \\ WWW: http://wWw. control.isy.liu.se \\ E-mail: gerdin@isy.liu.se
}

5th December 2005

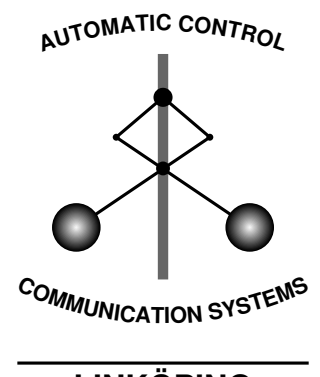

Report no.: LiTH-ISY-R-2711

Submitted to 14th IFAC Symposium on System Identification, SYSID-2006

Technical reports from the Control \& Communication group in Linköping are available at http://www.control.isy.liu.se/publications. 


\begin{abstract}
Identifiability is important to guarantee convergence in system identification applications, and observability is important in applications such as control and diagnosis. In this paper, recent results on analysis of nonlinear differentialalgebraic equations are used to derive criteria for local identifiability and local weak observability for such models. The criteria are based on rank tests. Examples show the relationship between the new criteria and standard methods for state-space systems.
\end{abstract}

Keywords: Identifiability, Observability, Nonlinear systems, Modelling, Identification, Descriptor systems 


\title{
LOCAL IDENTIFIABILITY AND \\ OBSERVABILITY OF NONLINEAR DIFFERENTIAL-ALGEBRAIC EQUATIONS ${ }^{1}$
}

\author{
Markus Gerdin \\ gerdin@isy.liu.se \\ Division of Automatic Control, Department of Electrical \\ Engineering, Linköping University, SE-581 83 Linköping, \\ Sweden
}

\begin{abstract}
Identifiability is important to guarantee convergence in system identification applications, and observability is important in applications such as control and diagnosis. In this paper, recent results on analysis of nonlinear differentialalgebraic equations are used to derive criteria for local identifiability and local weak observability for such models. The criteria are based on rank tests. Examples show the relationship between the new criteria and standard methods for statespace systems.
\end{abstract}

Keywords: Identifiability, Observability, Nonlinear systems, Modelling, Identification, Descriptor systems

\section{INTRODUCTION}

For nonlinear systems, the concepts of local identifiability and local weak observability are closely related. In this paper, the relation between the concepts is used to treat them in a common framework.

Identifiability of a model structure means that it is possible to estimate any unknown time-invariant parameters from measurements of the system. This is important for example if the unknown parameters represent physical parameters that are of interest. Numerical search methods may also have difficulties estimating the parameters if the model structure is not identifiable. References on identifiability are, e.g., Walter (1982) and Ljung (1999).

\footnotetext{
1 This work has been supported by the Swedish Foundation for Strategic Research (SSF) through VISIMOD and EXCEL and by the Swedish Research Council (VR) which is gratefully acknowledged. The author would also like to thank the reviewers for valuable comments.
}

Observability of a model means that it is possible to estimate any time-varying parameters using measurements from the system. Observability is important in many applications, for example control and diagnosis. Observability is treated in books on nonlinear control systems such as the one by Nijmeijer and van der Schaft (1990) and the one by Isidori (1989).

The model structure discussed in this paper is differential-algebraic equation (DAE) models, which is a quite general nonlinear model structure. A DAE model consists of a mixture of differential and algebraic equations. Differentialalgebraic equation models are also known as descriptor models and singular systems. Some references on the subject are (Dai, 1989; Brenan et al., 1996). The paper by Kunkel and Mehrmann (2001) discusses an analysis method for nonlinear DAE that will be the base for the results in the present paper. The interest to study DAE models partly comes from the fact that such models are generated by object-oriented modeling tools 
that have become more popular in recent years. An example of such a modeling tool is Modelica (Fritzson, 2004; Tiller, 2001).

\section{PRELIMINARIES}

In this section necessary background information on identifiability, observability and differentialalgebraic equations is presented.

\subsection{Differential-algebraic equations}

In general, a DAE can be written as

$$
F(\dot{x}(t), x(t), t)=0
$$

where $x(t) \in \mathbb{R}^{n}$ is a vector of internal timedependent variables, $F \in \mathbb{R}^{m}$, and $\dot{x}(t)=\frac{d x(t)}{d t}$. Furthermore, $t \in \mathbb{I}$ where $\mathbb{I} \subseteq \mathbb{R}$ is a compact interval. Note that $x(t)$ does not need to be a state vector, for example, the elements of $x(t)$ may not even appear differentiated in the equations. It is assumed that the system is solvable, otherwise it would me meaningless to discuss observability and identifiability. However, there may not exist solutions for all initial conditions $x(0)$. The initial conditions for which solutions exist will be denoted consistent initial conditions.

2.1.1. Analysis of DAE Since a nonlinear DAE is a general model structure, it can in many cases be difficult to analyze. For example, it is generally necessary to differentiate the equations a number of times (with respect to time) to find hidden relationships between the variables $x(t)$. Different methods have been proposed to formalize such differentiations. The results in this paper are based on (Kunkel and Mehrmann, 2001), so these results are summarized in this section. For proofs and a complete discussion, the reader is referred to (Kunkel and Mehrmann, 2001). These results are based on rank tests and the implicit function theorem, and are therefore only valid locally. For example, all ranks discussed below may depend on the current value of $x(t)$.

First define a nonlinear derivative array

$$
F_{l}\left(t, x, \dot{x}, \ldots, x^{l+1}\right)=0
$$

which stacks the original equations and all their derivatives up to level $l$ :

$$
F_{l}\left(t, x, \dot{x}, \ldots, x^{l+1}\right)=\left(\begin{array}{c}
F(\dot{x}, x, t) \\
\frac{d}{d t} F(\dot{x}, x, t) \\
\vdots \\
\frac{d^{l}}{d t^{l}} F(\dot{x}, x, t)
\end{array}\right)
$$

Partial derivatives of $F_{l}$ with respect to selected variables $p$ from $\left(t, x, \dot{x}, \ldots, x^{l+1}\right)$ are denoted by $F_{l ; p}$, e.g.

$$
F_{l ; \dot{x}, \ldots, x^{l+1}}=\left(\frac{\partial}{\partial \dot{x}} \cdots \frac{\partial^{l+1}}{\partial x^{l+1}}\right) F_{l} .
$$

A corresponding notation is used for partial derivatives of other functions.

The solution of the derivative array $F_{\mu}$ for some integer $\mu$ is denoted

$$
\mathbb{L}_{\mu}=\left\{z_{\mu} \in \mathbb{I} \times \mathbb{R}^{n} \times \cdots \times \mathbb{R}^{n} \mid F_{\mu}\left(z_{\mu}\right)=0\right\} .
$$

The following hypothesis, Hypothesis 1 by Kunkel and Mehrmann (2001), which describes the basic requirements on DAEs handled by the theory can now be formulated.

Hypothesis 1. Consider the general system of nonlinear DAEs (1). There exist integers $\mu, r, a, d$, and $v$ such that the following properties hold:

(1) The set $\mathbb{L}_{\mu} \subseteq \mathbb{R}^{(\mu+2) n+1}$ forms a manifold of dimension $(\mu+2) n+1-r$.

(2) We have

$$
\operatorname{rank} F_{\mu ; x, \dot{x}, \ldots, x^{(\mu+1)}}=r
$$

(3) We have

$$
\begin{aligned}
& \operatorname{corank} F_{\mu ; x, \dot{x}, \ldots, x^{(\mu+1)}-} \\
& \quad \operatorname{corank} F_{\mu-1 ; x, \dot{x}, \ldots, x(\mu)}=v
\end{aligned}
$$

on $\mathbb{L}_{\mu}$. (Note by current author: The corank is the rank deficiency with respect to rows. For example, if a matrix has 5 rows and rank 3 , the corank is 2.) The convention that corank $F_{-1, x}=0$ is used.

(4) We have

$$
\operatorname{rank} F_{\mu ; \dot{x}, \ldots, x^{(\mu+1)}}=r-a
$$

on $\mathbb{L}_{\mu}$ such that there are smooth full rank matrix functions $Z_{2}$ and $T_{2}$ defined on $\mathbb{L}_{\mu}$ of size $((\mu+1) m, a)$ and $(n, n-a)$, respectively, satisfying

$$
\begin{aligned}
Z_{2}^{T} F_{\mu ; \dot{x}, \ldots, x^{(\mu+1)}} & =0 \\
\operatorname{rank} Z_{2}^{T} F_{\mu ; x} & =a \\
Z_{2}^{T} F_{\mu ; x} T_{2} & =0
\end{aligned}
$$

on $\mathbb{L}_{\mu}$.

(5) We have

$$
\operatorname{rank} F_{\dot{x}} T_{2}=d=m-a-v
$$

on $\mathbb{L}_{\mu}$.

Using this hypothesis the following theorem, which is a reduced version of Theorem 3 by Kunkel and Mehrmann (2001), can be formulated.

Theorem 2. Let $F$ in (1) be sufficiently smooth and satisfy Hypothesis 1 with $\mu, r, a, d, v$. Then 
every solution of (1) locally solves a reduced problem,

$$
\begin{aligned}
& \dot{x}_{1}=\mathcal{L}\left(t, x_{1}, x_{2}, \dot{x}_{2}\right) \\
& x_{3}=\mathcal{R}\left(t, x_{1}, x_{2}\right)
\end{aligned}
$$

consisting of $d$ differential and $a$ algebraic equations. The elements of $x_{1} \in \mathbb{R}^{d}, x_{2} \in \mathbb{R}^{n-a-d}$, and $x_{3} \in \mathbb{R}^{a}$ together make up elements of $x$.

When using the method discussed in this section, it is normally necessary to successively increase $\mu$ until the hypothesis is true. The hypothesis could for example be verified by numeric rank tests at a certain value of $x(t)$, see further Remark 1 in (Kunkel and Mehrmann, 2001).

\subsection{Observability}

When discussing observability, a DAE with known inputs $u(t)$ and measured outputs $y(t)$ is considered:

$$
\begin{aligned}
G(\dot{z}(t), z(t), u(t), t) & =0 \\
y(t) & =h(z(t))
\end{aligned}
$$

The time-dependent internal variables are here denoted with $z(t)$. The internal variables may implicitly depend on derivatives of $u(t)$, so it will be assumed that the input is infinitely differentiable. This restriction of the input will generally not influence the observability of the system as defined here.

This contribution treats local weak observability, which means that the observability property only is examined locally. Formally, the following definitions are used to describe local weak observability. First, let the solution of the DAE (12) with the consistent initial condition $z_{0}$ and the input $u(t)$ be denoted $\pi\left(t ; z_{0}, u(t)\right)$. Two consistent initial conditions $z_{1}$ and $z_{2}$ are then indistinguishable if they give rise to the same output, i.e.,

$$
h\left(\pi\left(t ; z_{1}, u(t)\right)\right)=h\left(\pi\left(t ; z_{2}, u(t)\right)\right)
$$

for all infinitely differentiable $u(t)$. A natural definition of observability is then that if $z_{1}$ and $z_{2}$ are indistinguishable, then $z_{1}=z_{2}$. For local weak observability a more involved definition is necessary.

Definition 3. Let $U$ be an open set. Two consistent initial conditions $z_{1}$ and $z_{2}$ which both belong to $U$ are said to be $U$-indistinguishable if they give the same outputs in all cases where both trajectories lie entirely in $U$, i.e.,

$$
h\left(\pi\left(t ; z_{1}, u(t)\right)\right)=h\left(\pi\left(t ; z_{2}, u(t)\right)\right), t \in\left[t_{0}, t_{1}\right]
$$

as soon as

$$
\pi\left(t ; z_{1}, u(t)\right) \in U, \pi\left(t ; z_{2}, u(t)\right) \in U, t \in\left[t_{0}, t_{1}\right]
$$

for all infinitely differentiable inputs $u(t)$. The set of all points that is $U$-indistinguishable from $z_{0}$ is denoted $I_{U}\left(z_{0}\right)$.

It is now possible to give the definition of local weak observability:

Definition 4. The system (12) is locally weakly observable at the consistent initial condition $z_{0}$ if there exists an open neighborhood $U$ of $z_{0}$ such that for every neighborhood $V$ of $z_{0}$ with $V \subset U$, $I_{V}\left(z_{0}\right)=\left\{z_{0}\right\}$. If this is true for all points $z_{0}$, the system is locally weakly observable.

\subsection{Identifiability}

When discussing identifiability, a DAE model with a vector of unknown parameters $\theta$ is considered.

$$
\begin{aligned}
G(\dot{z}(t), z(t), \theta, u(t), t) & =0 \\
y(t) & =h(z(t))
\end{aligned}
$$

The parameters $\theta \in \mathbb{R}^{n_{\theta}}$ range over the set $D_{\mathcal{M}} \subseteq$ $\mathbb{R}^{n_{\theta}}$. Identifiability of a model structure means that if the output of two versions of the model are the same, then they have the same parameter vector $\theta$. The identifiability problem is considered without noise model, which is a common approach for nonlinear systems. Formally the following definition will be used. Similarly to the observability case, only local identifiability is treated.

Definition 5. The system (16) is locally identifiable at $\theta_{0}$ if there exists a neighborhood $V$ of $\theta_{0}$ such that

$$
\left.\begin{array}{c}
\theta \in V \\
y\left(\theta_{0}, t\right)=y(\theta, t)
\end{array}\right\} \Rightarrow \theta=\theta_{0}
$$

The system is locally identifiable if it is locally identifiable at all $\theta_{0} \in D_{\mathcal{M}}$.

\section{MAIN RESULTS}

Consider a nonlinear DAE with unknown parameters,

$$
\begin{aligned}
G(\dot{z}(t), z(t), \theta, u(t), t) & =0 \\
y(t) & =h(z(t))
\end{aligned}
$$

where $z(t) \in \mathbb{R}^{n_{z}}$ are internal variables, $\theta \in$ $\mathbb{R}^{n_{\theta}}$ unknown constant parameters, $u(t)$ external inputs, and $y(t)$ a measured output.

The idea when examining identifiability and observability for the system (18), is that if the system is observable and identifiable, then enough information should be contained in the equations to 
compute $z(t)$ and $\theta$ when $u(t)$ and $y(t)$ are known signals. This means that (18a) and (18b) booth should be used as equations that give information about $z(t)$ and $\theta$. However, if (18a) and (18b) are used as a system of differential-algebraic equations to compute $z(t)$ and $\theta$, the information that the parameters $\theta$ are constant must also be included. This can be expressed by the equation

$$
\dot{\theta}(t)=0 .
$$

The approach will thus be to examine if the equations (18a), (18b), and (19) together give enough information to compute $z(t)$ and $\theta$ locally. Collecting the equations gives the extended DAE

$$
\underbrace{\left(\begin{array}{c}
G(\dot{z}(t), z(t), \theta(t), u(t), t) \\
y(t)-h(z(t)) \\
\dot{\theta}(t)
\end{array}\right)}_{F}=0 .
$$

What needs to be done is to examine if $z(t)$ and $\theta$ can be solved uniquely from (20). It can be noted that a similar approach was used by Ljung and Glad (1994) to examine global identifiability for polynomial DAE using differential algebra. Locally, the uniqueness of the solutions can be examined using Theorem 2:

Theorem 6. Assume that the extended DAE (20) is sufficiently smooth and fulfills Hypothesis 1 for some $\mu, r, a, d$, and $v$ with

$$
x(t)=\left(\begin{array}{c}
z(t) \\
\theta(t)
\end{array}\right)
$$

at $z_{0}, \theta_{0}$. Then the original DAE (18) is locally identifiable and locally weakly observable at $z_{0}$, $\theta_{0}$ if and only if $a=n_{z}+n_{\theta}$.

Proof. Assume that $a=n_{z}+n_{\theta}$. Then, according to Theorem 2, the solution to the extended DAE (20) is locally described by

$$
x_{3}(t)=\mathcal{R}(t)
$$

and $x_{1}$ and $x_{2}$ have dimension 0 . Since $x_{3}$ then describes the solution for $z(t)$ and $\theta$, these variables are (locally) determined by the extended DAE. This means that if $y(t)$ is replaced by the output from a similar system with the parameter value $\theta_{0}$, then there is a neighborhood $V$ of $\theta_{0}$ where $\theta$ is uniquely determined by (20) so the original DAE is locally identifiable. The same holds for the initial condition, so the system is also locally weakly observable.

Now assume that $a<n_{z}+n_{\theta}$. Then, according to Theorem 2, the solution to the extended DAE (20) is locally described by

$$
\begin{aligned}
& \dot{x}_{1}(t)=\mathcal{L}\left(t, x_{1}(t), x_{2}(t), \dot{x}_{2}(t)\right) \\
& x_{3}(t)=\mathcal{R}\left(t, x_{1}(t), x_{2}(t)\right)
\end{aligned}
$$

where the dimension of at least one of $x_{1}$ and $x_{2}$ is greater than zero. This means that at least one of the $z(t)$ and/or $\theta$ has an undetermined initial condition or is a parameter that can be varied freely without changing the output $y(t)$. This means that the original system is either not locally weakly observable, not locally identifiable, or both.

Finally, the case $a>n_{z}+n_{\theta}$ cannot occur since $a$ is the difference between the ranks of the matrices in (6) and (8).

If it is known beforehand that the system is observable if all parameter values $\theta$ are known, then it is possible to examine local identifiability without having to treat observability at the same time. This is described in the following corollary.

Corollary \%. Assume that the original DAE (18) is locally weakly observable for known $\theta$ and that the extended DAE (20) fulfills Hypothesis 1 for some $\mu, r, a, d$, and $v$ with

$$
x(t)=\left(\begin{array}{c}
z(t) \\
\theta(t)
\end{array}\right)
$$

at $\theta_{0}$. Then the original DAE (18) is locally identifiable at $\theta_{0}$ if and only if $a=n_{z}+n_{\theta}$.

Proof. If $a=n_{z}+n_{\theta}$, the system is clearly both locally weakly observable and locally identifiable according to Theorem 6 . If $a<n_{z}+n_{\theta}$, the solution of the extended DAE is locally described by

$$
\begin{aligned}
& \dot{x}_{1}(t)=\mathcal{L}\left(t, x_{1}(t), x_{2}(t), \dot{x}_{2}(t)\right) \\
& x_{3}(t)=\mathcal{R}\left(t, x_{1}(t), x_{2}(t)\right) .
\end{aligned}
$$

Since $z(t)$ is locally weakly observable, it must be part of $x_{3}(t) . \theta$ is thus part of $x_{1}(t)$ and/or $x_{2}(t)$, so it is not locally identifiable.

If there are no unknown parameters, it may be interesting to examine observability by itself. In this case, the DAE is described by

$$
\begin{aligned}
G(\dot{z}(t), z(t), u(t), t) & =0 \\
y(t) & =h(z(t))
\end{aligned}
$$

and the extended DAE is

$$
\underbrace{\left(\begin{array}{c}
G(\dot{z}(t), z(t), u(t), t) \\
y(t)-h(z(t))
\end{array}\right)}_{F}=0 .
$$

A criterion for local weak observability is given by the following corollary.

Corollary 8. Assume that the extended DAE (27) fulfills Hypothesis 1 for some $\mu, r, a, d$, and $v$ with $x(t)=z(t)$ at $z_{0}$. Then the original DAE (26) is locally weakly observable at $z_{0}$ if and only if $a=n_{z}$. 
Proof. Follows directly from Theorem 6 with $n_{\theta}=$ 0 .

\section{EXAMPLES}

This section presents examples that show the relationship between the observability and identifiability criteria presented in this paper and other criteria in the literature.

Example 9. (Observability 1). In this example it is shown that for linear time-invariant state-space systems, the observability condition in Corollary 8 reduces to a standard observability test. Consider a linear state-space system,

$$
\begin{aligned}
& \dot{z}(t)=A z(t) \\
& y(t)=C z(t)
\end{aligned}
$$

where $z \in \mathbb{R}^{n_{z}}, n_{z} \geq 1$, and $y \in \mathbb{R}^{n_{y}}, n_{y} \geq 1$. The standard observability test that will be used is to check if the matrix

$$
\left(C^{T}(C A)^{T} \cdots\left(C A^{n_{z}}\right)^{T}\right)^{T}
$$

has full column rank.

The extended DAE is

$$
\underbrace{\left(\begin{array}{c}
\dot{z}(t)-A z(t) \\
C z(t)-y(t)
\end{array}\right)}_{F}=0,
$$

which gives the $\left(n_{z}+n_{y}\right)\left(n_{z}+1\right) \times\left(n_{z}+2\right) n_{z}$ matrix $F_{n_{z} ; x, \dot{x}, \ldots, x^{\left(n_{z}+1\right)}}($ with $x(t)=z(t))$

$$
F_{n_{z} ; x, \dot{x}, \ldots, x^{\left(n_{z}+1\right)}}=\left(\begin{array}{ccccc}
-A & I & 0 & \cdots & 0 \\
C & 0 & 0 & & \vdots \\
0 & -A & \ddots & 0 & 0 \\
\vdots & & 0 & -A & I \\
0 & \cdots & 0 & C & 0
\end{array}\right)
$$

Note that $m=n_{z}+n_{y}$ and $n=n_{z}$. Through row and column operations that do not change the matrix rank, $F_{n_{z} ; x, \dot{x}, \ldots, x^{\left(n_{z}+1\right)}}$ can be brought into the form

$$
\left(\begin{array}{ccccc}
0 & I & 0 & \cdots & 0 \\
C & 0 & 0 & & \vdots \\
0 & 0 & \ddots & 0 & 0 \\
\vdots & & 0 & 0 & I \\
C A^{n_{z}} & \cdots & 0 & 0 & 0
\end{array}\right)
$$

which has full column rank if and only if the standard observability (29) test is satisfied, so $F_{n_{z} ; x, \dot{x}, \ldots, x^{\left(n_{z}+1\right)}}$ has full column rank if and only if the standard observability test is satisfied.

To see how the transformation from (31) to (32) is done, consider the following example:

$$
\left(\begin{array}{ccc}
-A & I & 0 \\
C & 0 & 0 \\
0 & -A & I \\
0 & C & 0
\end{array}\right) \rightarrow\left(\begin{array}{ccc}
0 & I & 0 \\
C & 0 & 0 \\
-A^{2} & -A & I \\
A C & C & 0
\end{array}\right) \rightarrow\left(\begin{array}{ccc}
0 & I & 0 \\
C & 0 & 0 \\
0 & 0 & I \\
A C & 0 & 0
\end{array}\right)
$$

The first step is done by multiplying block column 2 by $A$ and adding to block column 1. Multiplying block row 1 by $A$ and adding to block row 3, multiplying block row 1 by $-C$ and adding to block row 4, and multiplying block column 3 by $A^{2}$ and adding to block column 1 gives the result.

It must now be shown that Hypothesis 1 is fulfilled for $\mu=n_{z}$ and the value of $a$ must be determined to see if the system is locally weakly observable.

First assume that $F_{n_{z} ; x, \dot{x}, \ldots, x^{\left(n_{z}+1\right)}}$ has full column rank so that $r=\left(n_{z}+2\right) n_{z}$. Then $v=\left(\left(n_{z}+\right.\right.$ $\left.\left.n_{y}\right)\left(n_{z}+1\right)-\left(n_{z}+2\right) n_{z}\right)-\left(\left(n_{z}+n_{y}\right) n_{z}-\right.$ $\left.\left(n_{z}+1\right) n_{z}\right)=n_{y}$. Furthermore, $a=n_{z}$ since $F_{n_{z} ; x, \dot{x}, \ldots, x^{\left(n_{z}+1\right)}}$ has full column rank. This gives that $T_{2}$ is the empty matrix, so $d=0$. Also, $m-a-v=0$, so Hypothesis 1 is satisfied with $a=n_{z}$, so the system is locally weakly observable according to Corollary 8 .

Now assume that $F_{n_{z} ; x, \dot{x}_{1, \ldots, x}\left(_{z}+1\right)}$ does not have full rank, so that $r+\Delta r=\left(n_{z}+2\right) n_{z}$ for some $\Delta r>0$. Then $v=\left(\left(n_{z}+n_{y}\right)\left(n_{z}+1\right)-\left(n_{z}+2\right) n_{z}+\right.$ $\Delta r)-\left(\left(n_{z}+n_{y}\right) n_{z}-\left(n_{z}+1\right) n_{z}+\Delta r\right)=n_{y}$. Now, $a=n_{z}-\Delta r$ since $F_{n_{z} ; \dot{x}, \ldots, x^{(n z+1)}}$ has full column rank by construction. Also, $d=\Delta r$ since $F_{\dot{x}}$ has full column rank by construction. This gives that $m-a-v=\Delta r$ so Hypothesis 1 is satisfied. Therefore Corollary 8 gives that the system is not locally weakly observable.

Example 10. (Observability 2). In this example it is discussed how the observability condition presented in this paper relates to a standard observability test for nonlinear state-space systems. Consider the nonlinear state-space system

$$
\begin{aligned}
& \dot{z}(t)=f(z(t)) \\
& y(t)=h(z(t))
\end{aligned}
$$

where $z \in \mathbb{R}^{n_{z}}, n_{z} \geq 1$, and $y \in \mathbb{R}^{n_{y}}, n_{y} \geq 1$.

The extended DAE is

$$
\underbrace{\left(\begin{array}{c}
\dot{z}(t)-f(z(t)) \\
h(z(t))-y(t)
\end{array}\right)}_{F}=0 .
$$

Note that the time derivatives of $h(z(t))$ can be defined recursively by

$$
\begin{aligned}
h^{(0)}(z(t)) & =h(z(t)) \\
h^{(i+1)}(z(t)) & =h_{z}^{(i)}(z(t)) f(z(t))
\end{aligned}
$$

and that the time derivatives of $f(z(t))$ can be defined recursively by

$$
\begin{aligned}
f^{(0)}(z(t)) & =f(z(t)) \\
f^{(i+1)}(z(t)) & =f_{z}^{(i)}(z(t)) f(z(t)) .
\end{aligned}
$$

This gives the $\left(n_{z}+n_{y}\right)(\mu+1) \times(\mu+2) n_{z}$ matrix $F_{\mu ; x, \dot{x}, \ldots, x(\mu+1)}($ with $x(t)=z(t))$ 


$$
F_{\mu ; x, \dot{x}, \ldots, x^{(\mu+1)}}=\left(\begin{array}{cccccc}
f_{z}(z(t)) & I & 0 & \cdots & 0 & 0 \\
h_{z}(z(t)) & 0 & 0 & & & \vdots \\
f_{z}^{(1)}(z(t)) & 0 & I & & & \vdots \\
h_{z}^{(1)}(z(t)) & 0 & 0 & \ddots & & \vdots \\
\vdots & & & & 0 & 0 \\
f_{z}^{(\mu)}(z(t)) & 0 & 0 & \cdots & 0 & I \\
h_{z}^{(\mu)}(z(t)) & 0 & 0 & \cdots & 0 & 0
\end{array}\right) .
$$

It is easy to see that this matrix has full column rank if and only if the matrix

$$
\left(\begin{array}{c}
h_{z}(z(t)) \\
h_{z}^{(1)}(z(t)) \\
\vdots \\
h_{z}^{(\mu)}(z(t))
\end{array}\right)
$$

has full column rank. Note that full column rank of this matrix for some $\mu$ is a (sufficient but not necessary) observability condition used by some authors.

Also, if Hypothesis 1 is satisfied for some $\mu, r, a$, $d$, and $v$, then since $F_{\mu ; \dot{x}, \ldots, x(\mu+1)}$ has full column rank by construction, $a$ equals the column rank of (37). Therefore, according to Corollary 8 the system is locally weakly observable if and only if (37) has full column rank for a $\mu$ that satisfies the hypothesis. Note that the condition is an if and only if-condition as long as Hypothesis 1 is satisfied. This is useful since rank conditions such as (37) for observability usually just give implications.

Example 11. (Identifiability). In this example it is shown how the criterion in Theorem 6 is related to a standard identifiability test for nonlinear statespace systems. Consider the state-space model

$$
\begin{aligned}
\dot{z}(t) & =f(z(t), \theta) \\
y(t) & =h(z(t)) .
\end{aligned}
$$

The extended DAE is

$$
\underbrace{\left(\begin{array}{c}
\dot{z}(t)-f(z(t), \theta(t)) \\
h(z(t))-y(t) \\
\dot{\theta}(t)
\end{array}\right)}_{F}=0 .
$$

Similarly to what was done in Example 10, it can be derived that if Hypothesis 1 is satisfied for some value of $\mu$, then the system is locally weakly observable and locally identifiable if and only if the matrix

$$
\left(\begin{array}{c}
h_{z, \theta}(z(t), \theta) \\
h_{z, \theta}^{(1)}(z(t), \theta) \\
\vdots \\
h_{z, \theta}^{(\mu)}(z(t), \theta)
\end{array}\right)
$$

has full column rank. This is a version of the identifiability test in Section 3.2.1 in (Walter, 1982) (use of the implicit function theorem to examine identifiability) if the so-called exhaustive summary is taken as the derivatives of the output.

\section{CONCLUSIONS}

In this paper, criteria for local identifiability and local weak observability of nonlinear differentialalgebraic equations have been derived using results by Kunkel and Mehrmann (2001). The inherent complexity of nonlinear differential-algebraic equations make the criteria somewhat involved, but on the other hand the generality of DAE models allows many systems to fit into the framework. For example, models generated by object-oriented modeling tools such as Modelica are specified as differential-algebraic equations.

Examples have shown that the criteria are closely related to standard identifiability and observability criteria for state-space systems.

\section{REFERENCES}

Brenan, K. E., S. L. Campbell and L. R. Petzold (1996). Numerical Solution of Initial-Value Problems in Differential-Algebraic Equations. Classics In Applied Mathematics. SIAM. Philadelphia.

Dai, L. (1989). Singular Control Systems. Lecture Notes in Control and Information Sciences. Springer-Verlag. Berlin, New York.

Fritzson, P. (2004). Principles of Object-Oriented Modeling and Simulation with Modelica 2.1. Wiley-IEEE. New York.

Isidori, A. (1989). Nonlinear Control Systems, An Introduction. Second ed. Springer-Verlag. Berlin, Heidelberg.

Kunkel, P. and V. Mehrmann (2001). Analysis of over- and underdetermined nonlinear differential-algebraic systems with application to nonlinear control problems. Mathematics of Control, Signals, and Systems 14(3), 233-256.

Ljung, L. (1999). System Identification - Theory for the User. Information and System Sciences Series. Second ed. Prentice Hall PTR. Upper Saddle River, N.J.

Ljung, L. and T. Glad (1994). On global identifiability for arbitrary model parametrizations. Automatica 30(2), 265-276.

Nijmeijer, H. and A. van der Schaft (1990). Nonlinear Dynamical Control Systems. SpringerVerlag. New York.

Tiller, M. (2001). Introduction to Physical Modeling with Modelica. Kluwer. Boston, Mass.

Walter, E. (1982). Identifiability of State Space Models with Applications to Transformation Systems. Vol. 46 of Lecture Notes in Biomathematics. Springer-Verlag. Berlin, Heidelberg, New York. 


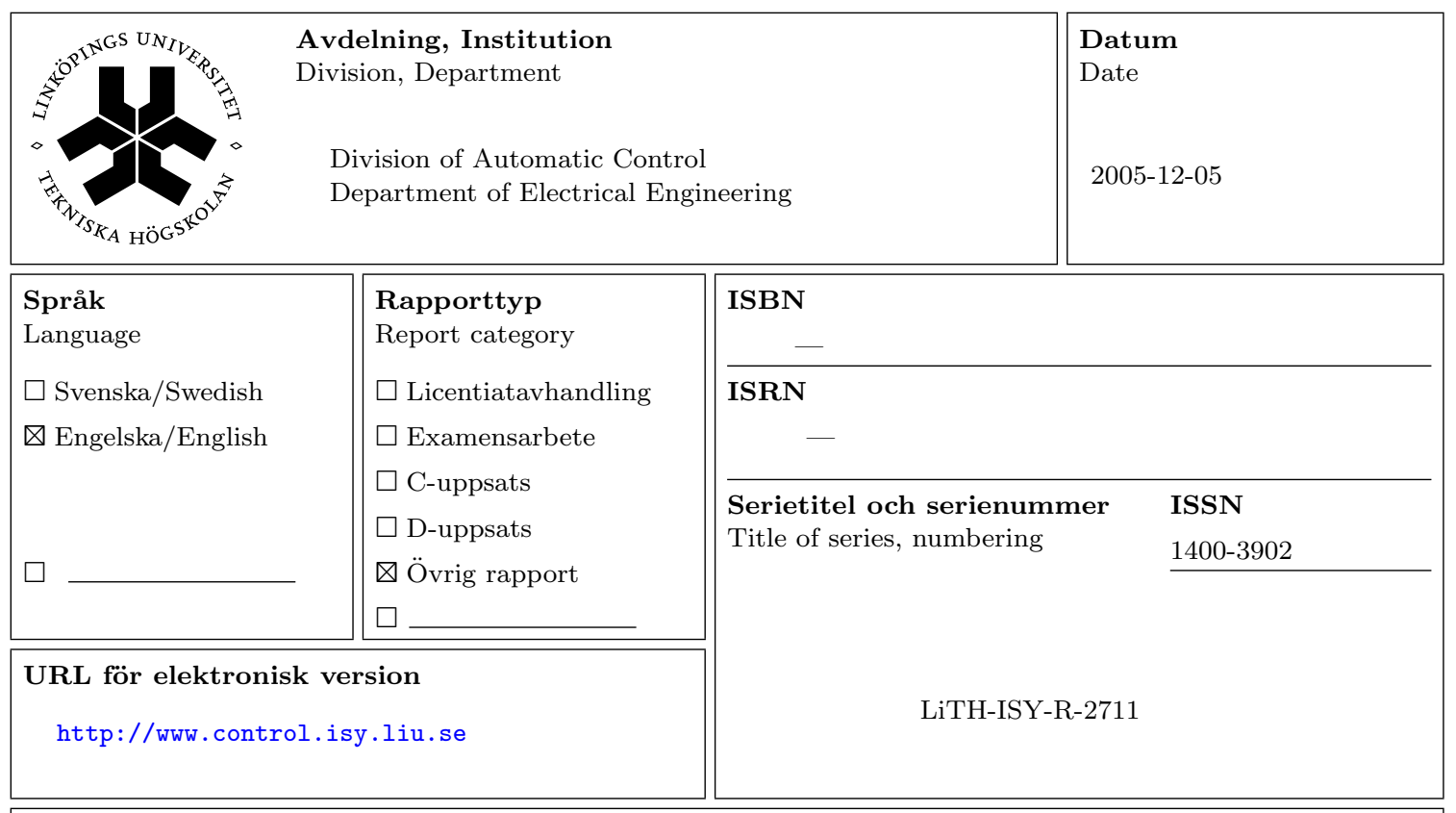

Titel Local Identifiability and Observability of Nonlinear Differential-Algebraic Equations

Title

Författare Markus Gerdin

Author

\section{Sammanfattning}

Abstract

Identifiability is important to guarantee convergence in system identification applications, and observability is important in applications such as control and diagnosis. In this paper, recent results on analysis of nonlinear differential-algebraic equations are used to derive criteria for local identifiability and local weak observability for such models. The criteria are based on rank tests. Examples show the relationship between the new criteria and standard methods for state-space systems. 